\title{
Mesures thermiques des flux de sève dans les troncs et les racines et fonctionnement hydrique des arbres. I. Analyse théorique des erreurs sur la mesure des flux et validation des mesures en présence de gradients thermiques extérieurs
}

\author{
B Cabibel, F Do \\ avec la participation technique de $\mathrm{J}$ Horoyan \\ INRA, station de science du sol, domaine Saint-Paul, BP 91, 84143 Montfavet cedex, France
}

(Reçu le 26 février 1990; accepté le 30 juin 1991)

\begin{abstract}
Résumé - Les flux de sève traversant des arbres fruitiers sont estimés par une méthode thermique. Basée sur la mesure des variations de température d'une source de chaleur chauffée à puissance constante et insérée dans le xylème des troncs et des racines de pommiers, elle est appliquée dans les conditions naturelles, à l'étude du comportement hydrique des arbres et de l'extraction de l'eau du sol par les racines. Une analyse théorique des erreurs sur la mesure des flux permet de déterminer le pourcentage de confiance obtenu sur l'estimation des flux de sève dans les 2 types d'organes considérés, et la contribution de chacune des variables du modèle de calcul à la formation de l'erreur totale. L'étude de l'effet des gradients thermiques extérieurs sur la mesure des flux, important notamment au niveau des racines, conduit à proposer un terme de correction de cette mesure. On montre que les mesures faites sur des arbres dont les caractéristiques morphologiques et végétatives sont représentatives des caractéristiques moyennes de l'ensemble des arbres d'un traitement considéré, sont représentatives à l'échelle du traitement ou de la parcelle si le traitement est uniforme.
\end{abstract}

Malus $=$ pommier $/$ tronc $/$ racine $/$ flux de sève $/$ erreur de mesure $/$ irrigation $/$ mesure thermique

Summary - Thermal measurements of sap flux in trunks and roots and hydrous behaviour of trees. I. Theoretical analysis of the errors in sap-flux estimations and measurement validation in the presence of external thermal gradients. A thermal method for estimating sap flow in apple-tree trunks and roots, based on measuring the temperature of a constant heat source inserted into the xylem had been applied for investigating water use by trees and soil-water uptake by roots under field conditions (figs 1,2). A theoretical analysis of the errors in flux measurement determined the percentage of confidence for the measurements of sap flux in 2 different types of organs studied (fig 3 and table I) and the contribution of each variable in the calculation model to the formation of the total error (table iI). The study of effect of the external thermal gradient on flux values (fig 4), especially important in the root zone, led us to propose a corrective term for this measurement (figs 5, 6; table III). Moreover, it has been shown that, for a given treatment, the flux measurements taken on trees with representative morphological and vegetative characteristics are representative at the level of the treatment or of the plot in the case of uniform treatment (fig 7; table IV).

Malus = apple tree $/$ trunk $/$ root $/$ sap flux $/$ measurement error $/$ irrigation / thermal measurement 


\section{INTRODUCTION}

La mesure des transferts hydriques dans les arbres fruitiers est expérimentalement difficile et onéreuse à mettre en œuvre. Elle apparait, de plus, souvent imparfaite quant à la valeur réelle et à la signification du paramètre mesuré, de par les limitations qui caractérisent les méthodes d'évaluation utilisées. II en est notamment ainsi qu'il s'agisse de définir ces transferts au travers d'une quantification :

- soit de la fonction puits racinaire par analyse du bilan de l'eau dans le sol;

- soit de la transpiration par évaluation de la perte en eau au-dessus du couvert végétal.

Ces approches, outre qu'elles ne permettent qu'une détermination indirecte des transferts dans la plante, posent respectivement le problème :

- soit de la représentativité au niveau parcellaire des mesures faites localement, une grande hétérogénéité pouvant exister dans le sol pour les paramètres mesurés;

- soit le problème de l'imprécision dans l'estimation de l'ETP et dans l'obtention des coefficients culturaux ainsi que de leur évolution dans le temps.

L'estimation, par voie thermique, des débits de sève brute dans les racines et dans le tronc, celui-ci étant la voie de passage obligé du transfert hydrique global, constitue un moyen opérationnel supplémentaire d'appréhender et de quantifier de façon directe :

- les cinétiques des fonctionnements hydriques de la plante entière et/ou des racines;

- la transpiration réelle, s'il est possible d'analyser simultanément le devenir des réserves en eau propres au végétal, en fonction des variations spatiales et/ou temporelles de la disponibilité de l'eau du sol et de la demande climatique.

Nous donnons, dans ce qui suit, des résultats concernant l'analyse d'ensemble de l'erreur faite sur la mesure des flux dans les troncs et les racines de pommiers et les conditions de validation des mesures de flux en présence de gradients thermiques extérieurs.

\section{MATÉRIEL ET MÉTHODES}

\section{Principe de la méthode}

Le flux est estimé par la mesure, en continu, des variations de température d'une sonde chauffante li- néaire, sous l'effet des variations du courant de sève, la sonde étant chauffée en continu sous puissance constante et insérée radialement dans le bois d'aubier (Granier, 1985). La température de cette sonde, maximale à flux nul, décroît lorsque la vitesse de sève augmente, une quantité de chaleur croissante étant alors évacuée par convection. D'une relation d'étalonnage liant la vitesse de sève à la variation de la température de la sonde chaude, relation établie au laboratoire pour l'ensemble bois - capteur étudié, on déduit le flux de sève.

Pour minimiser les effets des variations de la température ambiante sur l'ensemble bois-sonde, on mesure la variation des différences de température $\delta T$ entre la sonde chaude et une sonde de référence identique non chauffante, insérée en amont et de façon similaire dans le bois. Cette sonde de référence supposée en équilibre thermique avec le milieu boissève est sensée représenter la température au niveau de la sonde chauffée s'il n'y avait pas de chauffage.

\section{Mise en œuvre de la mesure}

Le capteur thermique utilisé est constitué de 2 sondes cylindriques de $2 \mathrm{~mm}$ de diamètre et de 1 ou $2 \mathrm{~cm}$ de longueur. Leur insertion radiale dans le bois conducteur, au-delà des assises corticales des racines ou des troncs, est réalisée légèrement en force de façon à obtenir le meilleur contact possible sonde-bois. Une protection thermique est installée à l'extérieur des sondes, aux points d'insertion dans les organes ligneux, pour minimiser les effets d'une variation brusque de la température extérieure sur les gradients thermiques existant au niveau de chacune des sondes (Do, 1986).

Pour éviter toute interférence thermique entre les 2 sondes, la sonde de référence est placée à une distance de 8-10 cm en amont de la sonde chaude et sur une génératrice légèrement décalée latéralement de façon à ne pas interrrompre les vaisseaux conduisant la sève jusqu'à la sonde supérieure (fig 1).

Les mesures sont faites en continu. Les tensions en provenance des capteurs sont enregistrées par une acquisition de données, disposant d'un pouvoir de résolution de $1 \mu \mathrm{V}$ (SAM 60A-AOIP) et pilotée par un logiciel implanté sur un microordinateur compatible PC. Le pas de mesure est variable. Il est modulé de 1 à $60 \mathrm{~min}$, selon le phénomène étudié.

Les résultats utilisés dans l'analyse de l'erreur faite sur la mesure des flux sont obtenus dans le tronc et

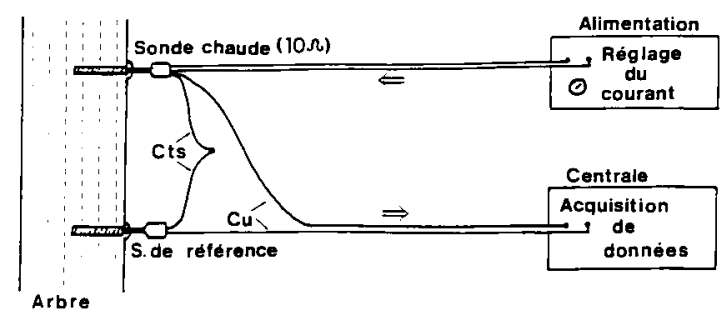

Fig 1. Dispositif de mesure du flux de sève. 
les racines d'un arbre isolé dans un verger expérimental, situé sur le domaine Saint-Paul (INRA, Monffavet). Planté dans les alluvions récentes argilo-calcaires de la Durance, il est constitué de pommiers Golden Delicious, greffés sur MM106, âgés de 8 ans. Les arbres sont plantés en quinconce selon une maille de $8 \times$ $5 \mathrm{~m}$. L'isolement de l'arbre étudié est réalisé par une tranchée périphérique creusée jusqu'à $2 \mathrm{~m}$ de profondeur et mise en place d'une barrière capillaire sous forme d'un film plastique afin d'isoler ce réservoir potentiel de tout prélèvement hydrique externe à l'arbre test.

Les résultats utilisés pour l'étude de la représentativité, au niveau parcellaire, des mesures de flux, faites sur des arbres tests, sont obtenus dans un verger de type industriel haie fruitière situé en centre expérimental horticole de Marsillargues (CEHM). Implanté depuis 1981, il est constitué de pommiers Golden Delicious greffés sur M9. Les arbres, conduits à axe vertical, sont distants de $1,5 \mathrm{~m}$ sur la raie de plantation, les rangs de $4 \mathrm{~m}$. Le sol est constitué d'alluvions récentes argilo-limoneuses calcaires. L'irrigation est de type aspersion.

\section{Estimation du flux}

Le flux de sève total $U$ est calculé à partir de la densité de flux Ju et de la surface de bois conductrice Sc, estimée dans le plan de la mesure (Granier, 1985).

$$
U=J u \cdot S c
$$

La densité de flux Ju est déduite de la relation d'étalonnage la liant à l'index de flux $K$ lui-même déterminé directement par mesure des variations de la température de la sonde chaude. La variable $K$, sans dimension, a pour expression:

$$
K=\frac{(T o-T b o)-(T u-T b)}{(T u-T b)}=\frac{\delta T o-\delta T u}{\delta T u}=\frac{\delta T o}{\delta T u}-1(2)
$$

avec : To = température de la sonde chaude à flux nul, Tbo = température du milieu bois-sève à flux nul; $T u=$ température de la sonde chaude à flux $U$, $T b=$ température du milieu bois-sève à flux $U$, $\delta T o=$ différence de température maximale à flux nul, $\delta T u=$ différence de température à flux $U$.

Pour une séquence de mesure de $n$ jours, l'estimation du terme $\delta$ To est réalisée, pour chaque sonde, en prenant l'enveloppe supérieure de la succession des maxima nocturnes de la période concernée.

La relation $K(J u)$, de type $K=a . J u^{\beta}$, est obtenue par ajustement non linéaire des couples de valeurs de $K$ et de $J u$, lors d'un étalonnage en laboratoire. Les densités de flux $J u$, traversant en régime permanent l'élément de bois à étudier, sont mesurées par pesée de l'exsudat. On donne en figure 2 la courbe d'étalonnage

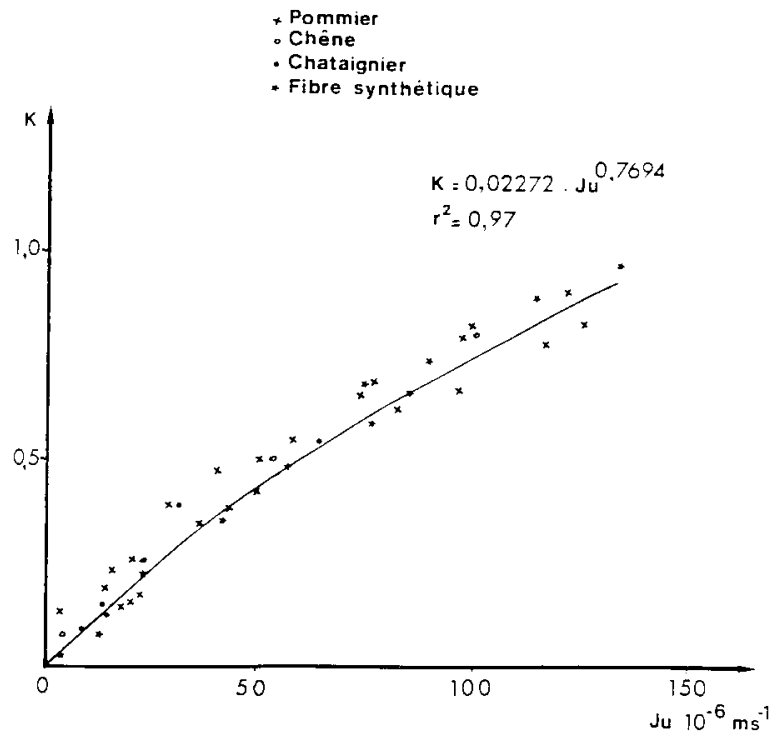

Fig 2. Relation expérimentale entre la densité de flux $J u$ et l'index de flux $K$ pour différentes espèces d'arbres.

établie pour des feuillus tests (Granier, 1985; Do, 1987).

La surface Sc est déterminée soit par carottage direct dans le tronc ou la racine à étudier, soit déduite de relations statistiques liant cette surface au diamètre de l'organe testé. L'expression finale du flux, selon que la surface conductrice est respectivement assimilable à un cercle ou à un anneau, devient :

$$
\begin{aligned}
& K^{1 / \beta} \quad K^{1 / \beta} \\
& U=[-] . \pi R^{2} \text { ou } U=[-] \quad . \pi\left(R^{2}-r^{2}\right) \\
& \alpha \\
& \alpha
\end{aligned}
$$

où $R$ et $r$ sont respectivement les rayons du bois d'aubier et du bois de cœur non conducteur, $\alpha$ et $\beta$ les coefficients d'ajustement de la relation $J u(K)$, obtenus en étalonnage.

\section{Expression des résultats}

Les flux sont exprimés soit:

- en densité de flux : ils sont alors exprimés en $\mathrm{ml} . \mathrm{h}^{-1} . \mathrm{cm}^{-2}$;

- en flux horaires : cette expression des flux, en $\mathrm{ml} . \mathrm{h}^{-1}$, qui nécessite l'estimation de l'aire de la section transversale de bois conducteur fonctionnel, est utilisée pour caractériser et comparer les cinétiques nycthémérales obtenues dans les différents organes étudiés.

- en flux cumulés sur la journée : exprimés en ml.j-1, ils correspondent pratiquement à la transpiration journalière. On les exprime également en $\mathrm{mm}^{-1}{ }^{-1}$, une surface au sol de 3,5 $\mathrm{m}^{2}$ étant par définition attribuée à chaque arbre. 


\section{Validation des mesures de flux}

\section{Analyse théorique de l'erreur sur la mesure des flux}

\section{Méthodologie utilisée}

Elle a consisté à décomposer la variance totale de $U$, le flux, au sens d'une dispersion de $\hat{u}$ estimée autour de $U$ réel, en analysant la contribution de chacune des variables du modèle de calcul, à savoir $\alpha, \beta, T o$, Tu et $R$ (Bruckler, 1983). En posant $Y=U$, en appelant $X_{i}$ toute variable de $U$ et en supposant que les $\sigma$ $\left(X_{i}\right)$ sont petits, la variance totale de $Y$ a pour expression (Dacunha-Castelle et Duflo, 1983) :

$$
\begin{aligned}
\sigma^{2}(Y) & =\sum_{i}\left[\frac{\delta Y}{\delta X_{i}}\right]^{2} \cdot \sigma^{2}\left(X_{i}\right) \\
& +\sum_{i \neq j}\left[\frac{\delta Y}{\delta X_{i}}\right]\left[\frac{\delta Y}{\delta X_{j}}\right] . \Gamma X_{i} X_{j}\left|\sigma\left(X_{i}\right) \cdot \sigma\left(X_{j}\right)\right|
\end{aligned}
$$

où :

$\sigma^{2}\left(X_{i}\right)$ : correspond à une dispersion expérimentale de $X_{i}$ autour de $X_{i}$ réel,

$\left(\delta Y / \delta X_{i}\right)^{2}$ : a le sens d'une sensibilité de $Y$ à une plus ou moins grande dispersion de la variable $X i$,

$\Gamma X_{i}, X_{j}$ : correspond à la corrélation entre les dispersions expérimentales de $X_{i}$ et $X_{i}$

Les limites maximales de l'erreur sur $R$, To et $T u$ étant déterminées à partir des mesures in situ (tableau I), les caractéristiques de $\alpha$ et $\beta$ étant déduites de l'étalonnage et la loi de distribution de û étant supposée suivre une loi normale, le développement de l'équation 4, pour l'ensemble des variables intervenant dans $Y$, permet d'estimer la variance totale $\sigma^{2}(U)$, la contribution relative de chacune des variables à la formation de l'erreur totale et les intervalles de confiance pour $U$ tels que :

$$
U \in[U-2 \sigma(U) ; U+2 \sigma(U)] \quad \text { à } 95 \%
$$

Tableau I. Bornes maximales de l'erreur déterminées in situ.

$\begin{array}{ccc}X i & 2 \sigma(X i) & \sigma(2 X I) \\ \alpha & 6,3.10^{-3} & 9,92.10^{-6} \\ \beta & 6,13.10^{-2} & 9,39.10^{-4} \\ (\alpha, \beta)=-0,993 & \text { (Uwhaus) } & \\ \sigma(T o) & \pm 5 \mu \mathrm{V} & 6,25 \\ \sigma(T u) & \pm 5 \mu \mathrm{V} & 6,25 \\ \text { (tronc) } & \pm 1,0 \mathrm{~mm} & 0,25 \\ \text { (racine) } & \pm 0,5 \mathrm{~mm} & 0,06\end{array}$

On donne l'incertitude relative IR sur la mesure des flux et la contribution des différentes sources d'erreur à la formation de l'erreur totale pour deux cas de figure caractéristiques, un tronc et une racine, et pour différentes valeurs de densité de flux de sève au tableau II. Le diagramme de l'évolution de $I R$ totale sur la mesure des flux dans les racines est donné en figure 3 .

\section{Structure de l'erreur et précision des mesures}

L'analyse des résultats fait apparaître essentiellement :

- 2 types de structure de l'erreur selon les termes considérés :

- pour $\alpha$ et $\beta$, ce sont les valeurs numériques élevées des sensibilités qui déterminent fortement leur contribution à l'erreur totale,

- pour $T o$, Tu et $R$, c'est l'amplitude de la variance expérimentale, $\sigma\left(X_{i}\right)$, qui détermine l'importance de cette contribution.

-3 zones de sensibilité différente quant à la précision des mesures de flux :

- une zone de mesure où la précision des mesures est très faible $( \pm 57 \%)$. Elle correspond à des flux très faibles $(J u \leq 2,5)$, flux estimés en période nocturne. La part prépondérante de l'erreur est liée aux termes constants $\alpha, \beta$ et $\delta$ To. On notera de plus que la composante relative à $T u$ doit y être par ailleurs surestimée, la dispersion de ce paramètre étant alors vraisemblablement inférieure à celle choisie,

- une zone où la précision des mesures est faible $4 \pm$ $23 \%)$. Elle correspond à des flux faibles $(2,5<J u<$ $10,0)$, flux qui apparaissent sur de très courtes périodes lors de régime de flux transitoire (apparition et disparition de l'ETP),

- une zone où la précision des mesures est bonne $( \pm 12 \%)$. Elle correspond aux flux moyens et forts

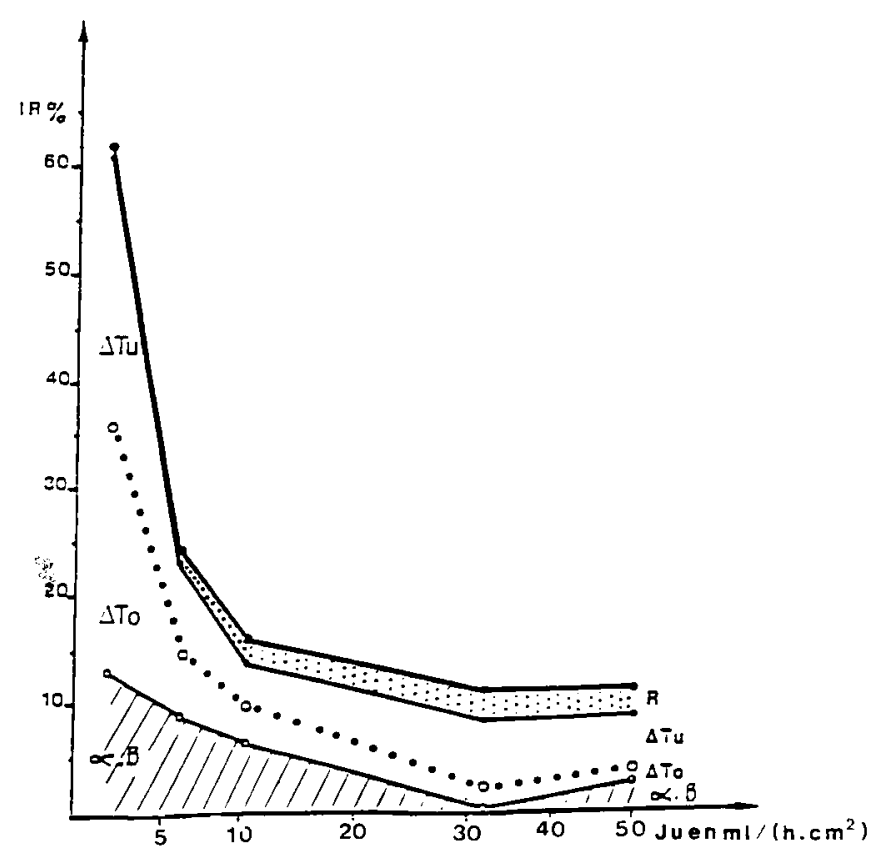

Fig 3. Incertitude relative et totale sur la mesure des densités de flux. 
Tableau II. Incertitude relative et contribution des diverses sources d'erreur à la formation de l'erreur totale.

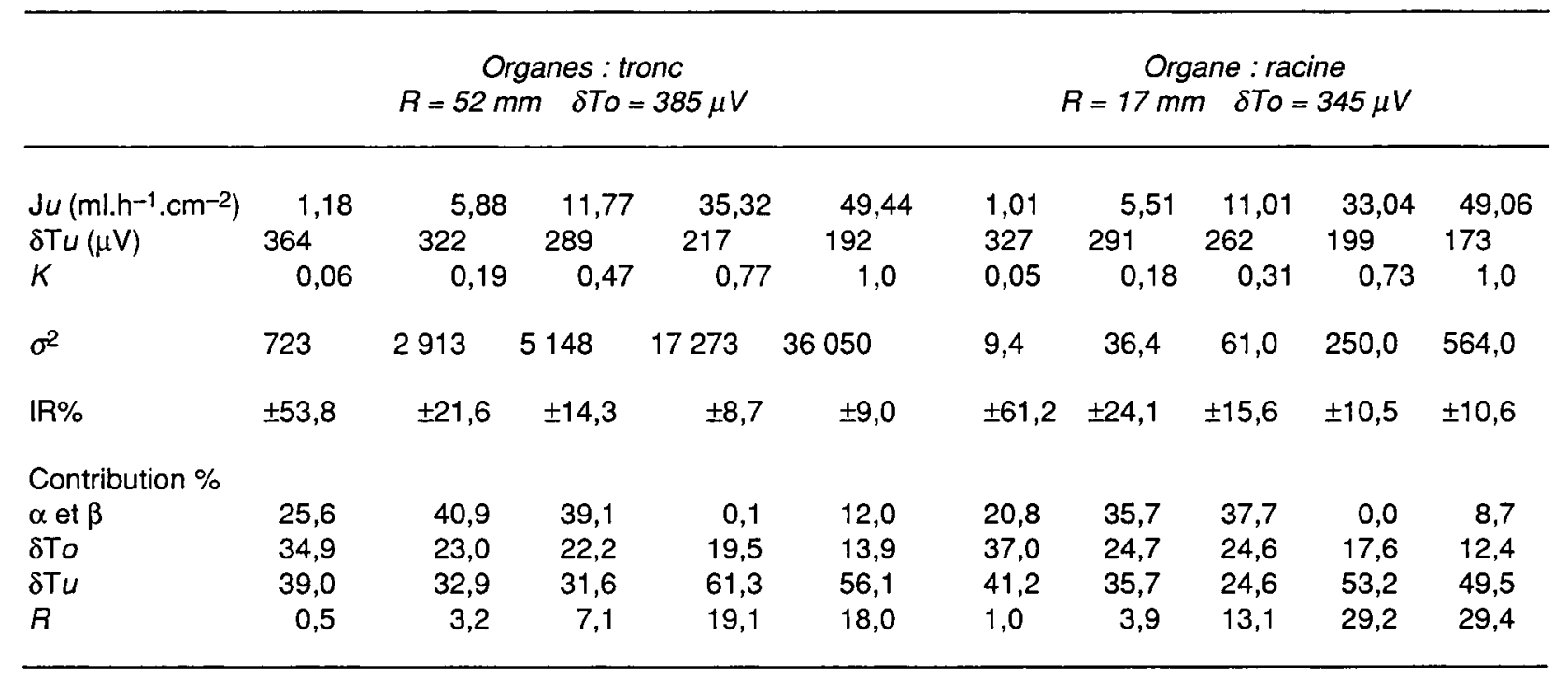

$(J u>10,0)$, précision qui entraîne, sur les bilans journaliers établis in situ, une précision du même ordre de grandeur que celle obtenue en conditions contrôlées (Claustre, 1987). Dans cette gamme de flux la part prépondérante de l'erreur est liée au terme Tu.

\section{Conclusion partielle}

La réduction de l'erreur sur la mesure des flux se ramène donc à la réduction des termes $\sigma^{2}(\alpha)$ et $\sigma^{2}(\beta)$ et à la réduction de la dispersion expérimentale sur $\delta$ To et $\delta T u$. Elle est donc liée à la qualité de l'étalonnage et implique une estimation de l'écart de température du milieu bois-sève, entre les 2 sondes et en l'absence de chauffage, sous l'effet d'un gradient de température extérieure.

\section{Analyse de l'effet d'un gradient thermique extérieur sur la mesure des flux}

\section{Hypothèse de base sur l'équilibre thermique du milieu bois-sève}

En posant, qu'en l'absence de chauffage, les températures du milieu bois-sève, Tbo et $T b$, températures mesurées par la sonde de référence, en l'absence et en présence de flux de sève, sont identiques dans les plans d'insertion des 2 sondes, l'index de flux $K^{\prime}$, index mesuré dans la pratique expérimentale, a pour expression :

$$
k^{\prime}=\frac{T o-T b o}{T u-T b}-1=\frac{\delta T o}{\delta T u}-1
$$

Or si cette condition sur Tbo et Tb est réalisée, en conditions thermiques isothermes, lors de l'étalonnage, l'expérience montre que l'ensemble de mesure, placé en conditions naturelles, peut être soumis à des interférences thermiques, notamment sous l'effet d'un gradient axial extérieur de température, et que, en l'absence de chauffage, les températures de la sonde chaude, $T^{\prime} b o$ et $T^{\prime} b$, peuvent être différentes de celles de la sonde de référence. En toute rigueur leurs expressions deviennent :

$$
T^{\prime} b o=T b o+d t o \text { et } T^{\prime} b=T b+\mathrm{d} t
$$

où les termes dto et $\mathrm{d} t$ sont les différences de températures induites par le gradient thermique extérieur, en l'absence de chauffage, entre les 2 sondes du capteur.

L'index de flux réel $K$ est donc égal à :

$$
\begin{aligned}
K & =\frac{T o-T^{\prime} b o}{T u-T^{\prime} b}-1=\frac{\delta T o+d t o}{\delta T u+d t}-1 \\
& =\frac{\delta T o(1+(\mathrm{d} t o / \delta T o))}{\delta T u(1+(\mathrm{d} t \delta T u))}
\end{aligned}
$$

ou exprimé en fonction de $K^{\prime}$ mesuré :

$$
K=\left(K^{\prime}+1\right) \frac{(1+(\mathrm{d} t o / \delta T o))}{(1+(d t / \delta T u))}-1
$$

Son estimation suppose donc la connaissance en continu des valeurs de dto et $\mathrm{d} t$.

\section{Estimation de $d$ to et $d \mathrm{t}$}

L'analyse a consisté à évaluer, dans les conditions exactes de l'expérimentation et pour chaque système capteur-bois, les régressions existantes entre les différences de température mesurées entre les 2 sondes, en l'absence de chauffage, et la variation des différences de températures entre celle du sol mesurée à $2 \mathrm{~cm}$ de profondeur et celle de l'air mesurée dans le plan de la sonde chaude, la sonde de référence étant très proche du sol. 
L'analyse des divers résultats obtenus montre que:

- les valeurs trouvées pour dto, valeurs apparaissant la nuit pour un $\delta$ To qui est la plus forte valeur des $\delta T u$ mesurés, sont toujours très faibles,

- l'amplitude de $\mathrm{d} t$, tant sur les troncs que sur les rameaux, est, pour un gradient thermique extérieur donné, d'autant plus grande que la distance capteur sol est faible et que la verticalité de l'organe testé est grande. Cet écart est, de plus, toujours plus important au niveau des racines excavées, notamment du fait de l'inversion des gradients thermiques air-sol pendant le jour et la nuit.

En conséquence, pour chaque capteur, on a établi la relation liant $\mathrm{d} t$ au gradient thermique extérieur (fig 4a), le programme de calcul corrigeant, à partir des températures extérieures mesurées, les valeurs de $K^{\prime}$ obtenues et les estimations réelles des flux (fig 5). En l'absence de données de température horaire et de mesure de rayonnement on a établi des relations liant $\mathrm{d} t$, en l'absence de chauffage, à l'ETP journalière (fig $4 b)$, qui permettent une correction des $K^{\prime}$ mesurés, à l'échelle de la journée.

\section{Effet théorique du gradient vertical de température extérieur sur la mesure de l'index de flux $\mathrm{K}$ et sur le sens de variation des estimations du flux}

Les valeurs de dto étant toujours très faibles permettent de négliger ce terme dans l'expression de $K$. L'index de flux réel devient donc égal à :

$$
K=\left(\left(K^{\prime}+1\right) /(1+(\mathrm{d} t \delta T u))\right)-1
$$

De l'expression de $K$ il ressort que si $d t$ est positif, $K$ est plus petit que $K^{\prime}$, ce dernier surestimant les va-
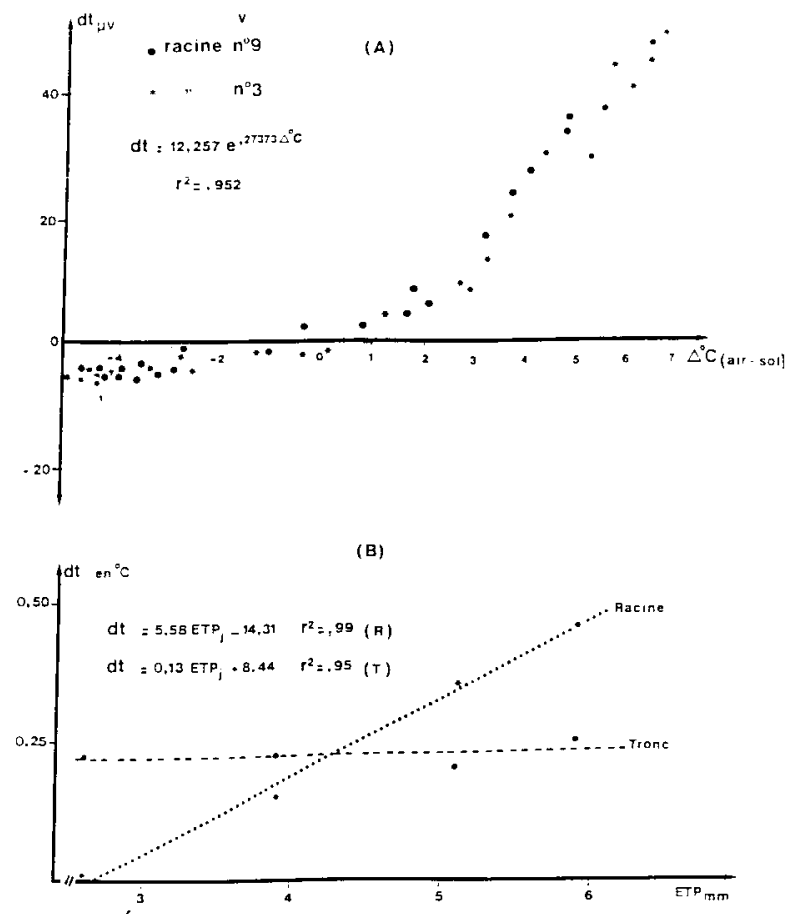

Fig 4. Écart de dt en fonction du gradient thermique extérieur (A) et de l'ETP (B). leurs de flux trouvées. Inversement si $d t$ est négatif, $K$ est supérieur à $K^{\prime}, K^{\prime}$ sousestimant les flux mesurés.

\section{Calcul du terme correctif de $\mathrm{K}^{\prime}$}

On a calculé un paramètre de correction de $K^{\prime}$, terme $B$ additif sur $\delta T o$, tel que $K^{\prime}=K$ réel :

$$
\frac{\delta T o+B}{\delta T u}-1=\frac{\delta T o / \delta T u}{1+(\mathrm{d} t / \delta T u)}-1
$$

Il ressort que :

$$
B=-\frac{\delta T o \cdot d t}{\delta T u+d t}
$$

\section{Validation du terme correctif B sur la mesure des flux}

On a comparé, sur une séquence de $5 \mathrm{j}$, les flux globaux journaliers mesurés dans le tronc à la somme des flux mesurés dans les 14 racines primaires d'un pommier. Exprimés en $\mathrm{mm}$, ces flux sont schématisés en figure 6, leurs amplitudes étant données en valeurs brutes et corrigées. On donne également, au tableau III les écarts relatifs entre les 2 types de flux mesurés avant et après correction.

L'estimation du fiux total journaiier au niveau du tronc, celui-ci étant soumis à des gradients thermiques extérieurs induisant un $\delta t$ faible et relativement constant, conduit après correction, à des valeurs de flux toujours supérieures mais proches des valeurs non corrigées. En conséquence la valeur corrigée "tronc" sera prise comme la valeur référence dans la comparaison avec les 2 types de flux obtenus dans les racines.

La somme des flux racinaires non corrigés apparaît très fortement sous-estimée par rapport au flux total mesuré dans le tronc, notamment aux fortes ETP. Ceci est en conformité avec les hypothèses précédemment émises sur le sens de variation de la dérive de l'estimation des flux sous l'effet d'un fort gradient extérieur de température. Inversement les valeurs corrigées pour les flux racinaires sont comprises dans l'intervalle de confiance estimé sur la mesure faite au niveau du tronc.

\section{Conclusion partielle}

La cohérence existant entre les valeurs de flux global et de la somme des flux racinaires corrigés ainsi que la parfaite concordance entre les cinétiques de chacun des types de flux par rapport à la contrainte climatique sont interprétées, en première approximation, comme une validation de l'utilisation du terme correctif $B$ dans le calcul des flux à partir des mesures brutes obtenues en expérimentation. Le calcul de $B$ nécessite néanmoins, simultanément aux mesures de flux, la mesure du gradient de température extérieure s'exerçant sur l'ensemble de mesure. 


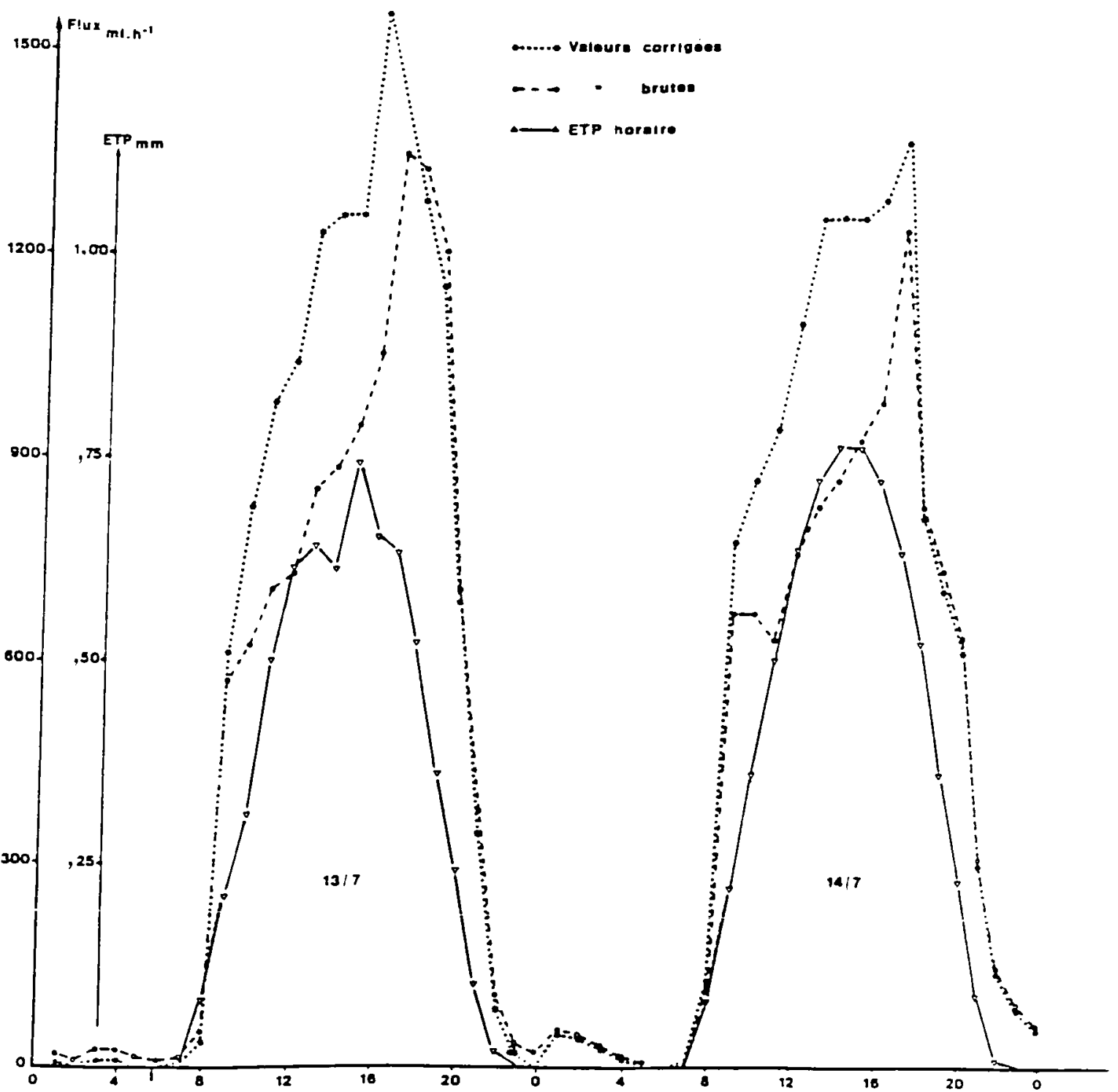

Fig 5. Valeurs des flux avant et après correction en fonction du gradient thermique extérieur.

\section{Représentativité parcellaire des mesures de flux}

Dans chaque traitement analysé, afin de s'assurer d'un minimum de représentativité des flux mesurés au niveau de la parcelle, on a :

- étudié, tant en cinétique qu'en amplitude, les flux horaires et journaliers traversant, sur une séquence de 45 j, 4 arbres répartis sur une raie de plantation,

- analysé statistiquement les écarts de flux obtenus pour toute la gamme des flux pouvant être rencontrés.

Les mesures présentées, caractéristiques du comportement des arbres, ont été faites entre le 9 et le 13 juillet 1989, dans le verger haie fruitière situé au CEHM de Marsillargues (Hérault). Les arbres tests choisis étaient ceux dont les caractéristiques morpho- logiques et végétatives (circonférence du tronc - développement végétatif et allongement des rameaux rendements antérieurs) étaient proches des caractéristiques moyennes des arbres du verger. Les cinétiques de flux horaires et l'analyse statistique des écarts de flux, tant horaires que journaliers, sont données en figure 7 et au tableau IV.

L'analyse des cinétiques de flux mises en évidence pour les 4 arbres tests, montre que, pour une contrainte climatique donnée et son évolution dans le temps à l'échelle de la journée ou de la séquence considérée, ces cinétiques de flux sont synchrones et en parfaite cohérence avec l'évolution de l'ETP enregistrée au CEHM.

Dans les 4 arbres tests, les flux peu élevés ou nuls hors demande climatique, subissent simultanément, avec l'apparition de cette dernière, une forte accéléra- 


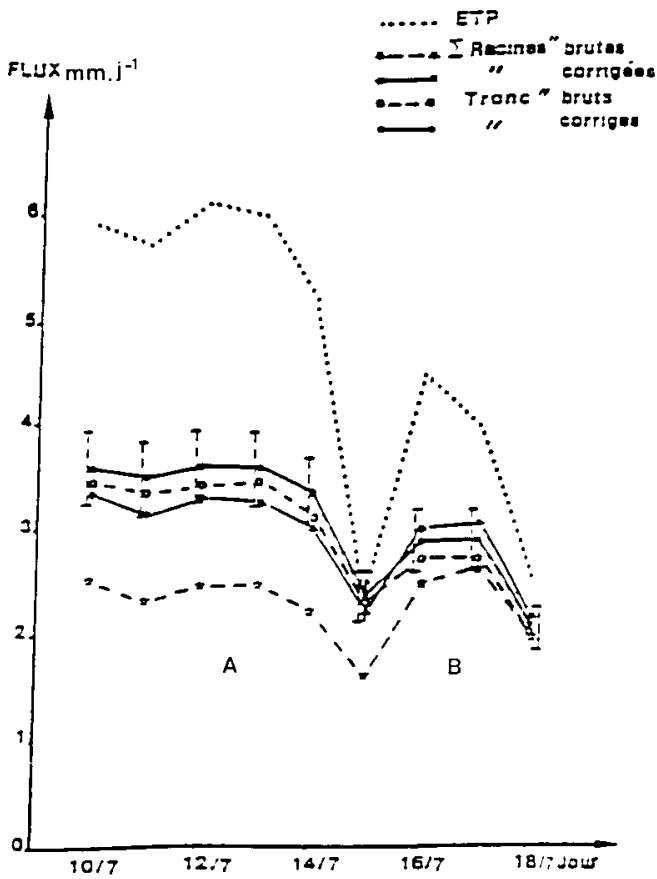

Fig 6. Flux journaliers $(\mathrm{mm} . j-1)$ dans le tronc et les racines en valeurs brutes et corrigées en période d'humectation du sol (A) et de réhumectation du sol (B).

tion jusqu'à des maxima se situant durant toute la période de forte ETP. Les flux décroissent par la suite avec elle, dans les 4 arbres considérés.

Il y a donc simultanéité des phénomènes instantanés caractérisant l'extraction de l'eau du sol par les racines de l'ensemble des arbres tests et des comportements hydriques des arbres.
L'analyse des écarts de mesures des flux horaires montre que l'incertitude relative totale sur la valeur des flux mesurés est fonction de l'intensité des flux. Les coefficients de variation calculés sont respectivement de $\pm 31,0 \%, \pm 33,5 \%, \pm 22,0 \%, \pm 15,2 \%$ et $\pm 5,3 \%$ pour des classes de flux égales à $0-70,70-250,250-$ $600,600-1000$ et supérieure à $1000 \mathrm{ml}^{-h^{-1}}$. L'incertitude relative atteint des valeurs élevées pour les flux très faibles et les flux existant lors de régimes transitoires, flux qui n'interviennent que pour une faible part dans l'estimation du flux global. Pour ce dernier l'incertitude relative totale est en moyenne de $\pm 5,9 \%$. L'ensemble des valeurs des coefficients de variation trouvées par classe de flux horaire et pour le flux global est donné au tableau IV.

\section{Conclusion partielle}

La mesure des flux hydriques, horaires et journaliers, dans les arbres fruitiers et leur évolution dans le temps, sont peu différentes dans des arbres ayant des caractéristiques morphologiques identiques et cela quelle que soit leur position dans la haie fruitière. $\mathrm{Si}$ les caractéristiques des arbres tests sont représentatives des caractéristiques morphologiques et végétatives moyennes de l'ensemble des arbres à l'échelle de la parcelle, il devient possible non seulement de mettre en évidence le comportement hydrique moyen du verger vis-à-vis de la demande climatique mais aussi de mesurer avec un intervalle de confiance de l'ordre de $\pm 10 \%$ les flux traversant les arbres.

\section{RÉSULTATS ET CONCLUSION}

La mesure des flux hydriques par voie thermique constitue un moyen, peu perturbant pour l'arbre,

Tableau III. Écarts relatifs de la somme des flux racinaires au flux mesuré dans le tronc en phases de dessèchement (A) et de réhumectation (B) du sol.

\begin{tabular}{|c|c|c|c|c|c|c|c|c|}
\hline \multirow[t]{2}{*}{ Date } & \multirow[t]{2}{*}{ Pluies } & ETP & \multirow{2}{*}{$\begin{array}{c}\text { Flux } \\
\text { total } \\
\text { tronc }\end{array}$} & \multicolumn{2}{|c|}{ Écart relatif } & $=$ & $\frac{(\Sigma R-T)}{T}$ & \multirow[b]{2}{*}{ Moy\% } \\
\hline & & $m m$ & & Brut\% & Моy\% & & Corrigé\% & \\
\hline 10 juill & 0,0 & 5,9 & 4,07 & $-27,2$ & & & $-6,3$ & \\
\hline 11 juill & 0,0 & 5,7 & 3,99 & $-31,1$ & & & $-11,2$ & \\
\hline 12 juill & 0,0 & 6,1 & 4,05 & $-28,4$ & $-29,4$ & & $-7,8$ & $-8,9$ \\
\hline 13 juill & 0,0 & 6,0 & 4,08 & $-29,7$ & & & $-9,7$ & \\
\hline 14 juill & 0,4 & 5,2 & 3,71 & $-30,5$ & (A) & & $-9,5$ & \\
\hline 15 juill & 5,4 & 2,4 & 2,72 & $-30,7$ & & & $-1,4$ & \\
\hline 16 juill & 36,5 & 4,5 & 3,22 & $-10,2$ & & & $+5,2$ & \\
\hline 17 juill & 13,1 & 4,0 & 3,23 & $-5,7$ & $-4,8$ & & $+6,0$ & $+4,9$ \\
\hline \multirow[t]{2}{*}{18 juill } & 0,2 & 2,5 & 2,32 & $+1,5$ & & & $+3,6$ & \\
\hline & & & & & (B) & & & \\
\hline
\end{tabular}




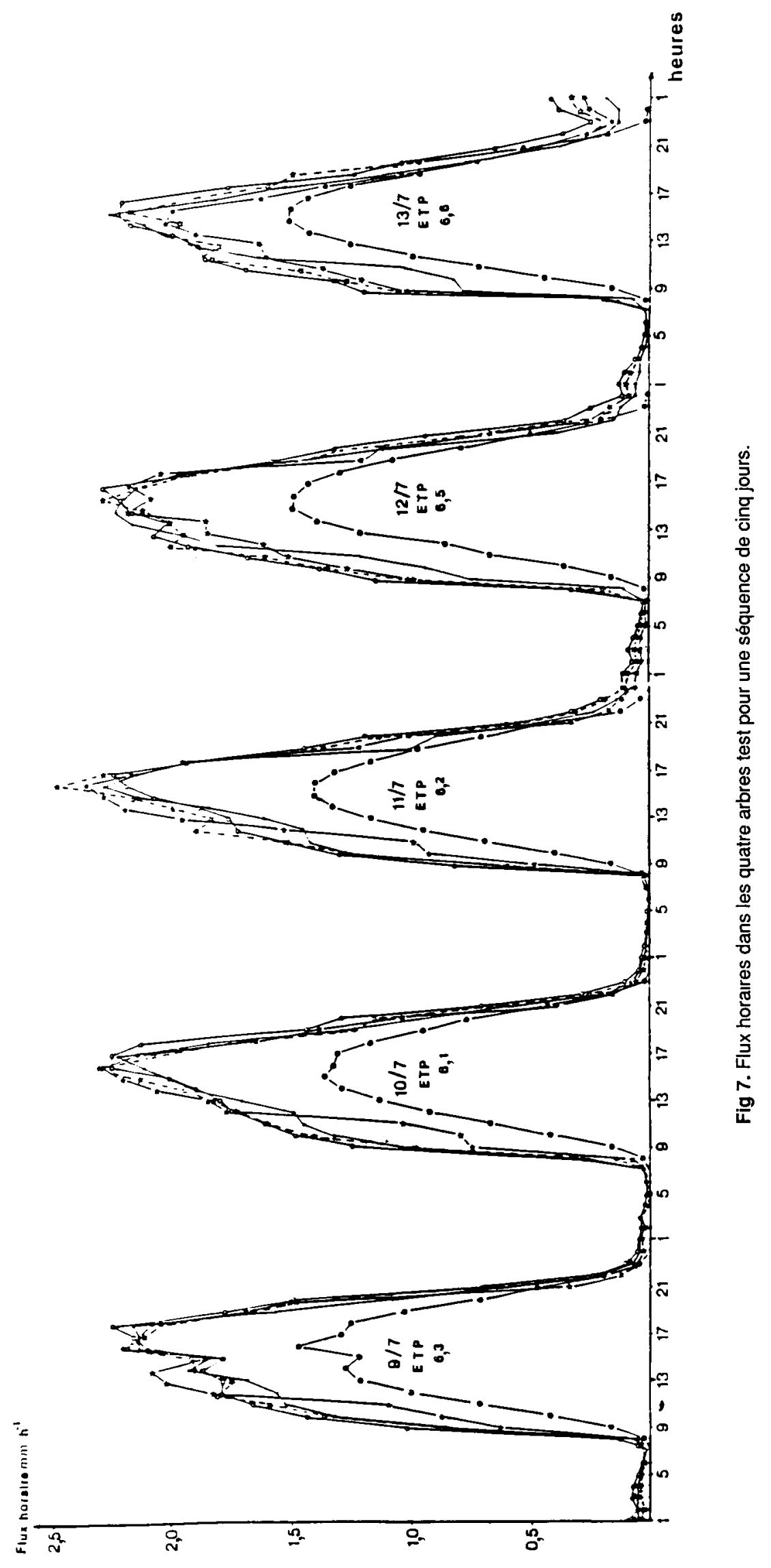


Tableau IV. Écarts moyens sur les flux horaires et journaliers mesurés dans les 4 arbres tests et coefficients de variation correspondants.

Flux horaires

\begin{tabular}{lccccc}
\hline$K$ & $K \leq 0,1$ & $0,1 K \leq 0,3$ & $0,3 K \leq \geq 0,6$ & $0,6 \leq K \geq 1,0$ & $1,0 \leq K$ \\
$U$ & $U \leq 70$ & $70 \leq U \geq 250$ & $250 \leq U \geq 550$ & $550 \leq U \geq 980$ & $980 \leq U$ \\
CV\% & $31,0 \pm 13,4$ & $33,5 \pm 9,1$ & $22,0 \pm 5,8$ & $15,2 \pm 4,3$ & $5,3 \pm 2,7$ \\
\hline
\end{tabular}

Flux journaliers

Date Flux moyen $\left(m l \dot{j}^{-1}\right) \quad$ CV\%

$\begin{array}{rrr}9 \text { juill } & 13000 \pm 580 & 4,46 \\ 10 \text { juill } & 12807 \pm 736 & 5,75 \\ 11 \text { juill } & 12462 \pm 640 & 5,14 \\ 12 \text { juill } & 13539 \pm 828 & 6,12 \\ 13 \text { juill } & 12447 \pm 1023 & 8,22 \\ & & \\ \text { moyen séquence } & 12851 \pm 761 & 5,94\end{array}$

permettant tout à la fois de caractériser son comportement hydrique en fonction de l'équilibre contrainte climatique - disponibilité de l'eau du sol et de quantifier, à une échelle de temps variable, l'extraction d'eau par les racines et son hétérogénéité spatiale.

Sa mise en œuvre nécessite néanmoins un étalonnage rigoureux de l'ensemble bois-capteur à étudier, une difficile détermination de l'aire conduisant la sève dans l'élément ligneux considéré et la prise en compte des interférences thermiques extérieures sur la réponse du capteur, dans les conditions de son utilisation in situ.

Si cet ensemble de données est contrôlé et que la mesure des flux est réalisée sur des individus, dont les caractéristiques morphologiques et végétatives sont représentatives de celles de l'ensemble des arbres du verger, il est possible de caractériser le comportement hydrique moyen du verger et de quantifier, avec une incertitude relative inférieure à $10 \%$, les flux horaires et journaliers.

\section{RÉFÉRENCES}

Bruckler L (1983) Analyse théorique des erreurs de mesure de teneur en eau et de la masse volumique par atténuation gamma. Bull GFHN 13, 7-33

Claustre JP (1987) Caractérisation du fonctionnement hydrique d'épiceas (Picea Abies $L$ ) en peuplement fermé : DEA de Biologie Forestière. CNRF - INRA Nancy

Dacunha-Castelle D, Duflo M (1983) Probablilité et Statistiques : deux problèmes à temps mobile. Masson, Paris

Do $F$ (1986) Caractérisation des flux racinaires et du comportement hydrique de pommiers sous irrigation localisée en sol fissuré. Mémoire de fin d'études, ENIT Bordeaux

Do F (1987) Caractérisation spatiale de l'extraction racinaire par la mesure des flux de sève brute en conditions intiales d'humectation homogène du sol. DEA option Science du sol ENSA Montpellier USTL Montpellier - INRA Montfavet

Granier A (1985) Une nouvelle méthode pour la mesure des flux de sève dans le tronc des arbres. Ann Sci For 42, 193-200 